九州大学学術情報リポジトリ

Kyushu University Institutional Repository

\title{
NONPARAMETRIC RECURSIVE KERNEL ESTIMATORS OF DISTRIBUTION FUNCTIONS
}

\section{Isogai, Eiichi}

Department of Mathematics, Faculty of Science, Niigata University

Hirose, Keiichi

Department of Mathematics, Faculty of Science, Niigata University

https://doi.org/10.5109/13435

出版情報: Bulletin of informatics and cybernetics. 26 (1/2), pp.87-99, 1994-03. Research Association of Statistical Sciences

バージョン：

権利関係 : 


\title{
NONPARAMETRIC RECURSIVE KERNEL ESTIMATORS OF DISTRIBUTION FUNCTIONS
}

\author{
By
}

\author{
Eiichi IsogaI* and Keiichi HIRos:*
}

\begin{abstract}
In this paper we propose nonparametric recursive kernel estimators for distribution functions and study their asymptotic properties, including strong uniform consistency. Rates of convergence of the mean square error are also investigated. Asymptotic normality of our estimators is shown.
\end{abstract}

\section{Introduction}

Let $X_{1}, X_{2}, \ldots$ be independent and identically distributed random variables having a common cumulative distribution function (cdf) $F$.

In this paper, we consider the problem of estimating the cdf $F$. Traditionally, as an estimator of $F$, the empirical distribution function was chosen. It is well known that the estimator is strongly uniformly consistent. On the other hand, alternative estimators of the cdf $F$ have been considered by (to mention a few) Nadaraya [6], Yamato [18], Winter [14, 15], Hill [4], Puri and Ralescu [7], Yukitch [19] and Sarda [10]. Especially, for kernel-type estimators, Singh et al. [11] investigated their asymptotic properties including strong uniform consistency and asymptotic normality, and also derived rates of convergence.

Under a certain condition on the cdf $F$, Read [8] showed that the empirical distribution function is inadmissible with respect to the mean integrated square error. Reiss [9] and Falk [2] showed that the kernel-type estimators have an asymptotically better performance on the level of deficiency than the empirical distribution function for appropriately chosen kernels and sufficiently smooth $F$.

By the way, the kernel-type estimators mentioned above are not recursive in nature, i.e. when the sample size increases, the estimators must be computed from the beginning. Besides, we are required to store extensive data in order to calculate them. With these things in mind, we propose a class of recursive kernel estimators $\left\{F_{n}\right\}$. By " recursive ", we mean that the estimator $F_{n}$ based on the first $n$ observations is a function of $F_{n-1}$ and the $n$th observation. Throughout this paper, the $\operatorname{cdf} F$ is assumed to be absolutely continuous with respect to Lebesgue measure, and let a probability density function (pdf) of $F$ be denoted by $f$.

\footnotetext{
* Department of Mathematics, Faculty of Science, Niigata University, Niigata 950-21, Japan
} 
In Section 2 we shall define a class of recursive kernel estimators of the cdf $F$. Section 3 gives consistency of the estimators. In Section 4 asymptotic expansions of the mean square error are derived. Also, our estimators are compared with the non-recursive kernel-type estimators from the viewpoint of the mean square error. In the last section we shall show the asymptotic normality of the estimators.

\section{Recursive kernel estimators}

In this section, we shall propose a class of recursive kernel estimators which is a modification of the kernel-type estimators.

Let $k$ (called a kernel) be a real-valued Borel measurable function on the real line $R$ such that

$$
M_{0} \equiv \int|k(y)| d y<\infty \text { and } \int k(y) d y=1,
$$

where throughout this paper all integrals are taken over $R$, unless otherwise specified. Let $\left\{h_{n}\right\}$ (called bandwidths) be a sequence of positive numbers converging to zero. For each $n \geq 1$, set

$$
k_{n}(y)=h_{n}^{-1} k\left(y / h_{n}\right), \quad K_{n}(x)=\int_{-\infty}^{x} k_{n}(y) d y, \quad K(x)=\int_{-\infty}^{x} k(y) d y
$$

and

$$
f_{n}(x)=n^{-1} \sum_{j=1}^{n} k_{j}\left(x-X_{j}\right)
$$

Now we define the recursive kernel estimators $\left\{F_{n}\right\}$ of the $\operatorname{cdf} F$ as

$$
F_{n}(x)=\int_{-\infty}^{x} f_{n}(y) d y=n^{-1} \sum_{j=1}^{n} K_{j}\left(x-X_{j}\right) \text { for } n \geq 1
$$

These estimators can be recursively computed as follows:

$$
F_{n}(x)=\left(1-n^{-1}\right) F_{n-1}(x)+n^{-1} K_{n}\left(x-X_{n}\right) \text { for } n \geq 1,
$$

where $F_{0}(x)=K(x)$.

REMARK 2.1. (i) Let $k$ be a pdf. Then $f_{n}(x)$ in $(2.1)$ and $F_{n}(x)$ in (2.2) are a $\mathrm{pdf}$ and a cdf, respectively. (ii) The non-recursive kernel-type estimators are defined by

$$
\hat{F}_{n}(x)=n^{-1} \sum_{j=1}^{n} K\left(\left(x-X_{j}\right) / h_{n}\right) \quad \text { for } n \geq 1 .
$$

(iii) As an estimator of the pdf $f$, Wolverton and Wagner [16], and Yamato [17] proposed the recursive kernel estimator $f_{n}(x)$ defined by $(2.1)$. 


\section{Consistency of the estimators}

In this section, the estimators $\left\{F_{n}\right\}$ defined by (2.2) are shown to be weakly consistent in quadratic mean and to be strongly uniformly consistent.

The following theorem gives weak consistency in quadratic mean.

THEOREM 3.1. The following relation holds true:

$$
\sup _{x} E\left[\left|F_{n}(x)-F(x)\right|^{2}\right] \rightarrow 0 \text { as } n \rightarrow \infty,
$$

where all suprema are taken over $R$ throughout this paper.

Proof. First of all, we shall prove

$$
\sup _{x}\left|E K_{n}\left(x-X_{n}\right)-F(x)\right| \rightarrow 0 \quad \text { as } n \rightarrow \infty .
$$

Fix any $x$. By Fubini's theorem, we have

$$
\left.E K_{n}\left(x-X_{n}\right)=\int_{-\infty}^{x}\left\{\int k(u) f\left(t-h_{n} u\right) d u\right)\right\} d t
$$

Hence, by the assumption on $k$, we get

$$
\begin{aligned}
& \sup _{x}\left|E K_{n}\left(x-X_{n}\right)-F(x)\right| \\
& =\sup _{x}\left|\int_{-\infty}^{x}\left\{\int k(u)\left(f\left(t-h_{n} u\right)-f(t)\right) d u\right\} d t\right| \\
& \leq \int\left\{\int\left|f\left(t-h_{n} u\right)-f(t)\right| d t\right\}|k(u)| d u .
\end{aligned}
$$

By using a theorem on the continuity of transformation of $L^{1}$-function (e.g. Wheeden and Zygmund [13, p.134]) and the convergence of $h_{n}$ to zero, we get that $\int \mid f\left(t-h_{n} u\right)-$ $f(t) \mid d t \rightarrow 0$ as $n \rightarrow \infty$ for each $u$. Thus, in view of the fact that $k, f \in L^{1}$ and the dominated convergence theorem, the right-hand side in (3.3) converges to zero. The relation (3.1) follows from this.

We shall now prove the theorem. Since

$$
\begin{aligned}
\sup _{x} E\left[K_{n}^{2}\left(x-X_{n}\right)\right] & =\sup _{x} \int\left\{\int_{-\infty}^{x} h_{n}^{-1} k\left((u-y) / h_{n}\right) d u\right\}^{2} f(y) d y \\
& \leq \int\left\{\int|k(t)| d t\right\}^{2} f(y) d y=M_{0}^{2},
\end{aligned}
$$

from (2.2) we get

$$
\sup _{x} \operatorname{Var}\left(F_{n}(x)\right) \leq n^{-2} \sum_{j=1}^{n} \sup _{x} E\left[K_{j}^{2}\left(x-X_{j}\right)\right] \leq M_{0}^{2} / n .
$$

On the other hand, by (3.1) and the fact that

$$
\sup _{x}\left|E F_{n}(x)-F(x)\right| \leq n^{-1} \sum_{j=1}^{n} \sup _{x}\left|E K_{j}\left(x-X_{j}\right)-F(x)\right|,
$$


we get

$$
\sup _{x}\left|E F_{n}(x)-F(x)\right| \rightarrow 0 \quad \text { as } n \rightarrow \infty
$$

Clearly

$$
\sup _{x} E\left[\left|F_{n}(x)-F(x)\right|^{2}\right] \leq \sup _{x} \operatorname{Var}\left(F_{n}(x)\right)+\left(\sup _{x}\left|E F_{n}(x)-F(x)\right|\right)^{2} .
$$

Thus, from (3.4) and (3.5), we obtain the theorem. dition.

We next consider strong uniform consistency. Here, we assume the following con-

Condition A. One of the following is true:

(i) The pdf $f$ is almost everywhere continuous, and $|x k(x)| \rightarrow 0$ as $|x| \rightarrow \infty$,

(ii) The pdf is bounded on $R$,

(iii) The kernel $k$ has a compact support,

(iv) $\int \varphi(x) d x<\infty$ with $\varphi(x) \equiv \sup _{|y| \geq|x|}|k(y)|$,

where the radial majorant $\varphi$ of $k$ is assumed to be measurable.

The following lemma can be easily proved by Theorems 2 and 3 of Devroye [1], Corollary C of Glick [3] and Theorem 2 of Stein [12, p.62].

Lemma 3.1. Assume Condition A. Let $k$ be a bounded pdf. Suppose

$$
\frac{n h_{n}}{\log \log n} \rightarrow \infty \quad \text { as } n \rightarrow \infty \quad \text { or } \quad \sum_{n=1}^{\infty}\left(n^{2} h_{n}\right)^{-1}<\infty \text {. }
$$

Then

$$
\int\left|f_{n}(x)-f(x)\right| d x \rightarrow 0 \quad \text { as } n \rightarrow \infty \quad \text { w.p.1. }
$$

We shall now give a theorem concerning strong uniform consistency.

Theorem 3.2. Assume Condition A. Let $k$ be a bounded pdf and $\left\{h_{n}\right\}$ satisfy (3.6). Then

$$
\sup _{x}\left|F_{n}(x)-F(x)\right| \rightarrow 0 \quad \text { as } n \rightarrow \infty \quad \text { w.p.1. }
$$

Proof. It is clear that

$$
\sup _{x}\left|F_{n}(x)-F(x)\right|=\sup _{x}\left|\int_{-\infty}^{x}\left(f_{n}(t)-f(t)\right) d t\right| \leq \int\left|f_{n}(t)-f(t)\right| d t,
$$

which, together with Lemma 3.1, yields the theorem.

\section{Asymptotic expansions of the estimators}

In this section, under the assumption that for an integer $r \geq 1$ the $r$ th derivative $f^{(r)}$ of $f$ is integrable, we shall give the asymptotic expansions of our estimators $F_{n}$. Similar 
results hold for $F_{n}$, provided that $f^{(r)}$ is bounded for an integer $r \geq 0$. We shall also compare our recursive estimators with the non-recursive kernel-type estimators given by (2.3) from the viewpoint of the mean square error.

As in Singh et al. [11], for each integer $r \geq 1$, let $\mathcal{K}_{r}$ be the class of all kernels $k$ such that

$$
\int y^{j} k(y) d y=\left\{\begin{array}{lll}
1 & \text { if } & j=0 \\
0 & \text { if } & j=1, \ldots, r-1
\end{array} \quad \text { and } \quad M_{r} \equiv \frac{1}{r !} \int\left|y^{r} k(y)\right| d y<\infty .\right.
$$

The following lemma provides the asymptotic behavior of the variance of $F_{n}$.

LEMMA 4.1. The following holds true: For each $x$,

$$
\operatorname{Var}\left(F_{n}(x)\right)=n^{-1} F(x)(1-F(x))+o\left(n^{-1}\right) \quad \text { as } n \rightarrow \infty .
$$

Proof. By (2.2), we get

$$
\operatorname{Var}\left(F_{n}(x)\right)=n^{-2} \sum_{j=1}^{n} E\left[K_{j}^{2}\left(x-X_{j}\right)\right]-n^{-2} \sum_{j=1}^{n}\left\{E K_{j}\left(x-X_{j}\right)\right\}^{2} .
$$

By using (3.1), we have

$$
n^{-1} \sum_{j=1}^{n}\left\{E K_{j}\left(x-X_{j}\right)\right\}^{2}=F^{2}(x)+o(1) \quad \text { as } n \rightarrow \infty
$$

Let $I(A)$ denote the indicator function of $A$. Since $K(x)=\int k(t) I(x>t) d t$, it follows from the assumption on $k$ that

$$
\begin{gathered}
\sup _{x}|K(x)| \leq M_{0}, \quad K(x) \rightarrow \begin{cases}1 & \text { as } x \rightarrow \infty \\
0 & \text { as } x \rightarrow-\infty,\end{cases} \\
K^{2}\left((x-y) / h_{j}\right) I(x>y) f(y) \rightarrow I(x>y) f(y) \text { as } j \rightarrow \infty
\end{gathered}
$$

and

$$
K^{2}\left((x-y) / h_{j}\right) I(x<y) f(y) \rightarrow 0 \quad \text { as } j \rightarrow \infty .
$$

Hence, by the dominated convergence theorem, we have

$$
\int K^{2}\left((x-y) / h_{j}\right) I(x>y) f(y) d y=F(x)+o(1) \quad \text { as } j \rightarrow \infty
$$

and

$$
\int K^{2}\left((x-y) / h_{j}\right) I(x<y) f(y) d y=o(1) \quad \text { as } j \rightarrow \infty
$$

which imply

$$
\begin{aligned}
& E\left[K_{j}^{2}\left(x-X_{j}\right)\right] \\
& =\int K^{2}\left((x-y) / h_{j}\right) I(x>y) f(y) d y+\int K^{2}\left((x-y) / h_{j}\right) I(x<y) f(y) d y \\
& =F(x)+o(1) \text { as } j \rightarrow \infty
\end{aligned}
$$


Thus

$$
n^{-1} \sum_{j=1}^{n} E\left[K_{j}^{2}\left(x-X_{j}\right)\right]=F(x)+o(1) \quad \text { as } n \rightarrow \infty .
$$

From (4.1)-(4.3), we obtain the lemma.

We shall give a uniform upper bound of the absolute value of the bias of $F_{n}$.

LEMMA 4.2. For an integer $r \geq 1$, let the rth derivative $f^{(r)}$ of $f$ be integrable. Let $k \in \mathcal{K}_{r}$. Then

$$
\sup _{x}\left|E F_{n}(x)-F(x)\right| \leq\left(n^{-1} \sum_{j=1}^{n} h_{j}^{r}\right) M_{r} \int\left|f^{(r)}(t)\right| d t .
$$

Proof. By (3.2), the Taylor expansion and the fact that $k \in \mathcal{K}_{r}$, we have

$$
\begin{aligned}
E & F_{n}(x) \\
= & n^{-1} \sum_{j=1}^{n} \int_{-\infty}^{x}\left\{\int k(u) f\left(t-h_{j} u\right) d u\right\} d t \\
= & n^{-1} \sum_{j=1}^{n} \int_{-\infty}^{x}\left\{\sum_{m=0}^{r-1} \frac{1}{m !} f^{(m)}(t)\left(-h_{j}\right)^{m} \int u^{m} k(u) d u\right. \\
& \left.+\frac{1}{(r-1) !} \int k(u)\left(\int_{t}^{t-h_{j} u}\left(t-h_{j} u-s\right)^{r-1} f^{(r)}(s) d s\right) d u\right\} d t \\
= & F(x) \\
& -n^{-1} \sum_{j=1}^{n} \frac{h_{j}^{r}}{(r-1) !} \int_{-\infty}^{x}\left[\int u^{r} k(u)\left\{\int_{0}^{1}(y-1)^{r-1} f^{(r)}\left(t-h_{j} u y\right) d y\right\} d u\right] d t .(4.4)
\end{aligned}
$$

Hence

$$
\begin{aligned}
& \sup _{x}\left|E F_{n}(x)-F(x)\right| \\
& \left.\leq n^{-1} \sum_{j=1}^{n} \frac{h_{j}^{r}}{(r-1) !} \iiint\left|u^{r} k(u)\right|\left\{\int_{0}^{1}|y-1|^{r-1}\left|f^{(r)}\left(t-h_{j} u y\right)\right| d y\right\} d u\right] d t \\
& =n^{-1} \sum_{j=1}^{n} \frac{h_{j}^{r}}{(r-1) !} \int\left|u^{r} k(u)\right| d u \int_{0}^{1}|y-1|^{r-1} d y \int\left|f^{(r)}(t)\right| d t \\
& =\left(n^{-1} \sum_{j=1}^{n} h_{j}^{r}\right) M_{r} \int\left|f^{(r)}(t)\right| d t
\end{aligned}
$$

which concludes the result of the lemma.

REMARK 4.1. We note that for $r \geq 3$ any fixed $k$ in $\mathcal{K}_{r}$ can be negative, thus leading to the negative estimator $F_{n}$ for the cdf $F$. But the bias $E\left[F_{n}(x)\right]-F(x)$ of 
$F_{n}(x)$ is reduced in absolute value. Hence, one can improve the rates of convergence of the bias to zero by using the kernel $k$ in $\mathcal{K}_{r}$.

From Lemma 4.2, (3.4) and the fact that

$$
E\left|F_{n}(x)-F(x)\right|^{2}=\operatorname{Var}\left(F_{n}(x)\right)+\left|E F_{n}(x)-F(x)\right|^{2},
$$

we can easily get the following result concerning a uniform upper bound of the mean square error of $F_{n}$.

Proposition 4.1. For an integer $r \geq 1$, let $f^{(r)}$ be integrable. Let $k \in \mathcal{K}_{r}$. Then

$$
\sup _{x} E\left|F_{n}(x)-F(x)\right|^{2} \leq M_{0}^{2} n^{-1}+\left(n^{-1} \sum_{j=1}^{n} h_{j}^{r}\right)^{2}\left(M_{r} \int\left|f^{(r)}(t)\right| d t\right)^{2} .
$$

We shall now give the exact asymptotic behavior of the bias of $F_{n}$.

Theorem 4.1. For an integer $r \geq 1$, let $f^{(r)}$ be intégrable. Let $k \in \mathcal{K}_{r}$ and $\left\{h_{n}\right\}$ satisfy

$$
\sum_{j=1}^{n} h_{j}^{r} \rightarrow \infty \quad \text { as } n \rightarrow \infty
$$

Then, as $n \rightarrow \infty$,

$$
E F_{n}(x)-F(x)=\left(n^{-1} \sum_{j=1}^{n} h_{j}^{r}\right)\left\{(-1)^{r} \mu_{r} \int_{-\infty}^{x} f^{(r)}(t) d t+o(1)\right\}
$$

uniformly in $x$, where

$$
\mu_{r}=\frac{1}{r !} \int y^{r} k(y) d y
$$

Proof. Set

$$
b_{j}=h_{j}^{r}, \quad B_{n}=\sum_{j=1}^{n} b_{j}, \quad a(x)=(-1)^{r} \mu_{r} \int_{-\infty}^{x} f^{(r)}(t) d t
$$

and

$$
a_{j}(x)=-\frac{1}{(r-1) !} \int_{-\infty}^{x}\left[\int u^{r} k(u)\left\{\int_{0}^{1}(y-1)^{r-1} f^{(r)}\left(t-h_{j} u y\right) d y\right\} d u\right] d t
$$

From (4.4) we get

$$
\left\{E F_{n}(x)-F(x)\right\} /\left(n^{-1} B_{n}\right)=B_{n}^{-1} \sum_{j=1}^{n} b_{j} a_{j}(x) .
$$

Since

$$
a(x)=-\frac{1}{(r-1) !} \int_{-\infty}^{x}\left[\int u^{r} k(u)\left\{\int_{0}^{1}(y-1)^{r-1} f^{(r)}(t) d y\right\} d u\right] d t
$$


we have

$$
\begin{aligned}
& \sup _{x}\left|a_{j}(x)-a(x)\right| \\
& \leq \frac{1}{(r-1) !} \int\left|u^{r} k(u)\right|\left[\int_{0}^{1}|y-1|^{r-1}\left\{\int\left|f^{(r)}\left(t-h_{j} u y\right)-f^{(r)}(t)\right| d t\right\} d y\right] d u \\
& \equiv \frac{1}{(r-1) !} I_{j}, \quad \text { say. }
\end{aligned}
$$

By virtue of the theorem on the continuity of transformation of $L^{1}$-function, the fact that $f^{(r)} \in L^{1}$ and the dominated convergence theorem, we have

$$
\int_{0}^{1}|y-1|^{r-1}\left\{\int\left|f^{(r)}\left(t-h_{j} u y\right)-f^{(r)}(t)\right| d t\right\} d y \rightarrow 0 \quad \text { as } j \rightarrow \infty
$$

for each $u$. Thus, by using the fact that $f^{(r)} \in L^{1}$ and $u^{r} k(u) \in L^{1}$ and the dominated convergence theorem, we have $I_{j} \rightarrow 0$ as $j \rightarrow \infty$, which, together with (4.6), yields

$$
\sup _{x}\left|a_{j}(x)-a(x)\right| \rightarrow 0 \quad \text { as } j \rightarrow \infty \text {. }
$$

Clearly

$$
\sup _{x}\left|B_{n}^{-1} \sum_{j=1}^{n} b_{j} a_{j}(x)-a(x)\right| \leq B_{n}^{-1} \sum_{j=1}^{n} b_{j} \sup _{x}\left|a_{j}(x)-a(x)\right| .
$$

Since $B_{n} \uparrow \infty$ as $n \rightarrow \infty$ by the assumption made, in view of this inequality, Toeplitz's lemma (see Loève [5, p.250]), (4.5) and (4.7), we obtain the result of the theorem.

By virtue of Lemma 4.1 and Theorem 4.1, we have the following corollary concerning the exact asymptotic behavior of the mean square error of $F_{n}(x)$.

Corollary 4.1. Assume all the conditions of Theorem 4.1. Then, for each $x$, as $n \rightarrow \infty$,

$$
\begin{aligned}
& E\left|F_{n}(x)-F(x)\right|^{2} \\
& =n^{-1} F(x)(1-F(x))+\left(n^{-1} \sum_{j=1}^{n} h_{j}^{r}\right)^{2}\left(\mu_{r} \int_{-\infty}^{x} f^{(r)}(t) d t\right)^{2} \\
& \quad+o\left(n^{-1}+\left(n^{-1} \sum_{j=1}^{n} h_{j}^{r}\right)^{2}\right) .
\end{aligned}
$$

Let $r \geq 0$ be an integer. Then, under the assumption that $f^{(r)}$ is bounded, we can obtain similar results. Let $k \in \mathcal{K}_{r+1}$. Then, by the Taylor expansion, we have the following result instead of (4.4):

$$
\begin{aligned}
& E F_{n}(x) \\
& =F(x)+n^{-1} \sum_{j=1}^{n} \frac{1}{(r+1) !}\left(-h_{j}\right)^{r+1} \int u^{r+1} k(u) f^{(r)}\left(x-\theta h_{j} u\right) d u
\end{aligned}
$$


with $0<\theta<1$.

The four results below can be proved by the same manner as above, so we omit the proofs.

Lemma 4.3. For an integer $r \geq 0$, let $f^{(r)}$ be bounded. Let $k \in \mathcal{K}_{r+1}$. Then

$$
\sup _{x}\left|E F_{n}(x)-F(x)\right| \leq\left(n^{-1} \sum_{j=1}^{n} h_{j}^{r+1}\right) M_{r+1}\left\|f^{(r)}\right\|,
$$

where $\left\|f^{(r)}\right\| \equiv \sup _{x}\left|f^{(r)}(x)\right|$.

Proposition 4.2. For an integer $r \geq 0$, let $f^{(r)}$ be bounded. Let $k \in \mathcal{K}_{r+1}$. Then

$$
\sup _{x} E\left|F_{n}(x)-F(x)\right|^{2} \leq M_{0}^{2} n^{-1}+\left(n^{-1} \sum_{j=1}^{n} h_{j}^{r+1}\right)^{2}\left(M_{r+1}\left\|f^{(r)}\right\|\right)^{2} .
$$

THEOREM 4.2. For an integer $r \geq 0$, let $f^{(r)}$ be bounded. Let $k \in \mathcal{K}_{r+1}$ and $\left\{h_{n}\right\}$ satisfy

$$
\sum_{j=1}^{n} h_{j}^{r+1} \rightarrow \infty \quad \text { as } n \rightarrow \infty
$$

Then, for each continuity point $x$ of $f^{(r)}$, as $n \rightarrow \infty$,

$$
E F_{n}(x)-F(x)=\left(n^{-1} \sum_{j=1}^{n} h_{j}^{r+1}\right)\left\{(-1)^{r+1} \mu_{r+1} f^{(r)}(x)+o(1)\right\}
$$

where

$$
\mu_{r+1}=\frac{1}{(r+1) !} \int y^{r+1} k(y) d y .
$$

If $f^{(r)}$ is uniformly continuous, then (4.8) holds uniformly in $x$.

CoRollary 4.2. Assume all the conditions of Theorem 4.2. Then, for each continuity point $x$ of $f^{(r)}$, as $n \rightarrow \infty$,

$$
\begin{aligned}
& E\left|F_{n}(x)-F(x)\right|^{2} \\
& =n^{-1} F(x)(1-F(x))+\left(n^{-1} \sum_{j=1}^{n} h_{j}^{r+1}\right)^{2}\left(\mu_{r+1} f^{(r)}(x)\right)^{2} \\
& \quad+o\left(n^{-1}+\left(n^{-1} \sum_{j=1}^{n} h_{j}^{r+1}\right)^{2}\right)
\end{aligned}
$$

We shall here compare our recursive estimators $\left\{F_{n}\right\}$ with the non-recursive estimators $\left\{\hat{F}_{n}\right\}$ given by (2.3) in terms of the mean square error. For an integer $r \geq 1$, let $f^{(r)}$ be integrable and $k \in \mathcal{K}_{r}$. We consider the following popular choice of $h_{n}$ : 


$$
h_{n}=n^{-\alpha} \text { with } 0<\alpha<r^{-1} \text {. }
$$

Set

$$
a(x)=F(x)(1-F(x)) \quad \text { and } \quad b(x)=\left(\mu_{r} \int_{-\infty}^{x} f^{(r)}(t) d t\right)^{2}
$$

From Corollary 4.1 , we have, as $n \rightarrow \infty$,

$$
\begin{aligned}
\operatorname{MSE}\left(F_{n}(x)\right) & \equiv E\left|F_{n}(x)-F(x)\right|^{2} \\
& =a(x) n^{-1}+b(x)(1-\alpha r)^{-2} n^{-2 \alpha r}+o\left(n^{-1}+n^{-2 \alpha r}\right) .
\end{aligned}
$$

On the other hand, we have from Corollary 4 of Singh et al. [11] that as $n \rightarrow \infty$,

$$
\operatorname{MSE}\left(\hat{F}_{n}(x)\right)=a(x) n^{-1}+b(x) n^{-2 \alpha r}+o\left(n^{-1}+n^{-2 \alpha r}\right) .
$$

Let us find the value of $\alpha$ which minimizes each mean square error. It is easy to see that for a fixed but sufficiently large value of $n$ the mean square error of $F_{n}(x)$ attains the minimum at $\alpha=r^{-1}-(r \log n)^{-1}$ while the mean square error of $\hat{F}_{n}(x)$ attains the minimum at $\alpha=r^{-1}$ when $0<\alpha \leq r^{-1}$. Now, as an $\alpha$ in the neighborhood of $r^{-1}$, we choose an arbitrary $\alpha$ with $(2 r)^{-1}<\alpha<r^{-1}$. Then, for any $x$ with $0<F(x)<1$, we obtain that $\lim _{n \rightarrow \infty} \operatorname{MSE}\left(F_{n}(x)\right) / M S E\left(\hat{F}_{n}(x)\right)=1$. Thus, in this case, $F_{n}(x)$ is asymptotically equivalent to $\hat{F}_{n}(x)$ in the sense of the mean square error.

\section{Asymptotic normality of the estimators}

In this section, we shall show the asymptotic normality of the estimators $\left\{F_{n}\right\}$.

THEOREM 5.1. For an integer $r \geq 1$, let $f^{(r)}$ be integrable. Let $k \in \mathcal{K}_{r}$ and $\left\{h_{n}\right\}$ satisfy

$$
n^{-\frac{1}{2}} \sum_{j=1}^{n} h_{j}^{r} \rightarrow 0 \quad \text { as } n \rightarrow \infty
$$

Then

$$
n^{\frac{1}{2}}\left(F_{n}(x)-F(x)\right) \stackrel{L}{\longrightarrow} N(0, F(x)(1-F(x))) \text { as } n \rightarrow \infty
$$

for each $x$ with $0<F(x)<1$, where " $\stackrel{L}{\longrightarrow}$ " stands for convergence in law.

Proof. Clearly

$$
\begin{aligned}
n^{\frac{1}{2}} & \left(F_{n}(x)-F(x)\right) /(F(x)(1-F(x)))^{\frac{1}{2}} \\
= & \left\{\left(F_{n}(x)-E F_{n}(x)\right) /\left(\operatorname{Var}\left(F_{n}(x)\right)\right)^{\frac{1}{2}}\right\}\left\{n \operatorname{Var}\left(F_{n}(x)\right) /(F(x)(1-F(x)))\right\}^{\frac{1}{2}} \\
& +n^{\frac{1}{2}}\left(E F_{n}(x)-F(x)\right) /(F(x)(1-F(x)))^{\frac{1}{2}} .
\end{aligned}
$$

From Lemma 4.2 and the assumption made, the second term of the right-hand side of (5.1) converges to zero as $n$ tends to infinity. Lemma 4.1 implies that

$$
\left\{n \operatorname{Var}\left(F_{n}(x)\right) /(F(x)(1-F(x)))\right\}^{\frac{1}{2}} \rightarrow 1 \text { as } n \rightarrow \infty .
$$


Hence, taking (5.1) into account, it suffices to show

$$
\left(F_{n}(x)-E F_{n}(x)\right) /\left(\operatorname{Var}\left(F_{n}(x)\right)\right)^{\frac{1}{2}} \stackrel{L}{\longrightarrow} N(0,1) \text { as } n \rightarrow \infty .
$$

Set $V_{n}=K_{n}\left(x-X_{n}\right)-E K_{n}\left(x-X_{n}\right)$ for $n=1,2, \ldots$. Then, $V_{1}, V_{2}, \ldots$ are independent but not identically distributed random variables; $E V_{n}=0$ for $n=1,2, \ldots$ It is clear that

$$
\left(F_{n}(x)-E F_{n}(x)\right) /\left(\operatorname{Var}\left(F_{n}(x)\right)\right)^{\frac{1}{2}}=\sum_{j=1}^{n} V_{j} /\left(\operatorname{Var}\left(\sum_{j=1}^{n} V_{j}\right)\right)^{\frac{1}{2}}
$$

From Lemma 4.1, we have

$$
n^{-1} \operatorname{Var}\left(\sum_{j=1}^{n} V_{j}\right)=F(x)(1-F(x))+o(1) \quad \text { as } n \rightarrow \infty .
$$

Since $\left|K_{j}\left(x-X_{j}\right)\right| \leq M_{0}$ for $j=1, \ldots, n$, we get

$$
E\left[\left|K_{j}\left(x-X_{j}\right)\right|^{3}\right] \leq M_{0}^{3} \text { for } j=1, \ldots, n \text {. }
$$

Hence, by Hölder's inequality and (5.4), we have, as $n \rightarrow \infty$,

$$
\begin{aligned}
\sum_{j=1}^{n} E\left[\left|V_{j}\right|^{3}\right] /\left(\operatorname{Var}\left(\sum_{j=1}^{n} V_{j}\right)\right)^{\frac{3}{2}} & \leq 8 \sum_{j=1}^{n} E\left[\left|K_{j}\left(x-X_{j}\right)\right|^{3}\right] /\left(\operatorname{Var}\left(\sum_{j=1}^{n} V_{j}\right)\right)^{\frac{3}{2}} \\
& \leq\left\{8 M_{0}^{3} /(F(x)(1-F(x))+o(1))^{\frac{3}{2}}\right\} n^{-\frac{1}{2}}
\end{aligned}
$$

which implies

$$
\sum_{j=1}^{n} E\left[\left|V_{j}\right|^{3}\right] /\left(\operatorname{Var}\left(\sum_{j=1}^{n} V_{j}\right)\right)^{\frac{3}{2}} \rightarrow 0 \text { as } n \rightarrow \infty
$$

Thus, by Lyapounov's theorem and (5.3), (5.2) is established. This completes the proof.

The following theorem can be proved in the same manner as Theorem 5.1 by using Lemma 4.3 .

TheOREM 5.2. For an integer $r \geq 0$, let $f^{(r)}$ be bounded. Let $k \in \mathcal{K}_{r+1}$ and $\left\{h_{n}\right\}$ satisfy

$$
n^{-\frac{1}{2}} \sum_{j=1}^{n} h_{j}^{r+1} \rightarrow 0 \quad \text { as } n \rightarrow \infty
$$

Then

$$
n^{\frac{1}{2}}\left(F_{n}(x)-F(x)\right) \stackrel{L}{\longrightarrow} N(0, F(x)(1-F(x))) \quad \text { as } n \rightarrow \infty
$$

for each $x$ with $0<F(x)<1$. 


\section{References}

[1] Devroye, L. : On the pointwise and the integral convergence of recursive kernel estimates of probability densities, Utilitas Math., 15 (1979), 113-128.

[2] Falk, M. : Relative efficiency and deficiency of kernel type estimators of smooth distribution function, Statist Neerlandica, 37 (1983), 73-83.

[3] Glick, N. : Consistency conditions for probability estimators and integrals of density estimators, Utilitas Math., 6 (1974), 61-74.

[4] Hill, P. D. : Kernel estimation of a distribution function, Comm. Statist. - Theory Meth., 14 (1985), 605-620.

[5] Loève, M. : Probability Theory, Vol. I, 4th ed., Springer, New York, 1977.

[6] Nadaraya, E. A. : Some new estimates for distribution functions, Theory Probab. Appl., 9 (1964), 497-500.

[7] Puri, M. L. and Ralescu, S. S. : Central limit theorem for perturbed empirical distribution functions evaluated at a random point, J. Multivariate Anal., 19 (1986), 273-279.

[8] Read, R. R. : The asymptotic inadmissibility of the sample distribution function, Ann. Math. Statist., 43 (1972), 89-95.

[9] Reiss, R. D. : Nonparametric estimation of smooth distribution functions, Scand. J. Statist., 8 (1981), 116-119.

[10] Sarda, P. : Smoothing parameter selection for smooth distribution functions, $J$. Statist. Plann. Inference, 35 (1993), 65-75.

[11] Singh, R. S., Gasser, T. and Prasad, B. : Nonparametric estimates of distribution functions, Comm. Statist. - Theory Meth., 12 (1983), 2095-2108.

[12] Stein, E. M. : Singular Integrals and Differentiability Properties of Functions, Princeton Univ. Press, Princeton, New Jersey, 1970.

[13] Wheeden, R. L. and Zygmund, A. : Measure and Integral, Marcel Dekker, Inc., New York and Basel, 1977.

[14] Winter, B. B. : Strong uniform consistency of integrals of density estimators, Canad. J. Statist., 1 (1973), 247-253.

[15] Winter B. B. : Convergence rates of perturbed empirical distribution functions, $J$. Appl. Probab., 16 (1979), 163-173.

[16] Wolverton, C. T. and Wagner, T. J. : Asymptotically optimal discriminant function for pattern classification, IEEE Trans. Inform. Theory, IT-15 (1969), 258-265. 
[17] Yamato, H. : Sequential estimation of a continuous probability density function and mode, Bull. Math. Statist., 14 (1971), 1-12.

[18] Yamato, H. : Uniform convergence of an estimator of a distribution function, Bull. Math. Statist., 15 (1973), 69-78.

[19] Yukitch, J. E. : A note on limit theorems for perturbed empirical processes, Stochastic Process. Appl., 33 (1989), 163-173.

Received October 20, 1993

Revised January 10, 1994

Communicated by H. Yamato 